\title{
Relación de las Medidas de Mecánica Ventilatoria y Radiográficas con el Tiempo de Conexión a Ventilación Mecánica en Pacientes COVID-19. Un Análisis Preliminar
}

\author{
Relations of Ventilatory Mechanical and Radiographic Measures with the Connection \\ Time to Mechanical Ventilation in Patients COVID-19: A Preliminary Analysis
}

\author{
Rodrigo Muñoz Cofrée ${ }^{1,2,3}$; Patricio Araneda Madrid ${ }^{3}$; Mariano del Sol ${ }^{1,2}$; Felipe Álvarez Pérez ${ }^{3}$; \\ Camilo Pérez Riquelme ${ }^{3}$; Máximo Escobar Cabello ${ }^{4,5}$ \& Jorge Valenzuela Vásquez ${ }^{3}$
}

\begin{abstract}
MUÑOZ-COFRÉ, R.; ARANEDA-MADRID, P.; DEL SOL, M.; ÁLVAREZ-PÉREZ, F.; PÉREZRIQUELME, C.; ESCOBARCABELLO, M. \& VALENZUELA-VÁSQUEZ, J. Relación de las medidas de mecánica ventilatoria y radiográficas con el tiempo de conexión a ventilación mecánica en pacientes COVID-19. Un análisis preliminar. Int. J. Morphol., 38(6):1580-1585, 2020.
\end{abstract}

RESUMEN: Los pulmones son el sitio predominante en la infección por COVID-19. Esta puede conducir al síndrome distrés respiratorio agudo (SDRA). Frente a su sintomatología severa, la ventilación mecánica (VM), y sus valores de mecánica ventilatoria aparecen como una herramienta fundamental. Un complemento, para analizar el estado de avance de esta patología es la radiografía de tórax (RT), aunque en ocasiones esta depende de la experiencia del equipo de salud. Así el objetivo de esta investigación fue explorar la relación de las medidas de mecánica ventilatoria y radiográficas con el tiempo de conexión a VM en pacientes COVID-19. Estudio retrospectivo, que incluyó a 23 pacientes en VM. Se recolectó información de variables de mecánica ventilatoria; PEEP, presión plateau, presión de distensión y compliance estática. Desde la RT se midió, altura y ancho pulmonar, ángulo costodiafragmático y espacio intercostal. Los resultados indicaron que las variables de mecánica ventilatoria tales como el PEEP y el plateau se relacionaron significativamente con el tiempo de conexión a VM $(\mathrm{r}=0,449 ; \mathrm{p}=0,035$ y $\mathrm{r}=0,472 ; \mathrm{p}=0,026)$, mientras que las variables radiográficas construidas en base al ángulo costodiafragmático y el espacio intercostal presentaron similares comportamientos $(\mathrm{r}=0,462 ; \mathrm{p}=0,046 \mathrm{y}$ $\mathrm{r}=-0,543 ; \mathrm{p}=0,009)$. En conclusión, la presión resultante de la programación del ventilador mecánico junto a cambios estructurales observados en la RT, se relacionan con el tiempo de conexión a VM.

PALABRAS CLAVE; COVID-19; Radiografía de tórax; Ventilación mecánica.

\section{INTRODUCCIÓN}

El COVID-19 es una enfermedad causada por el virus SARS-CoV-2, perteneciente a la familia de los Coronavirus. En diciembre del año 2019, la Organización Mundial de Salud (OMS) en China alertó sobre pacientes con neumonía de etiología desconocida (Ministerio de Salud, 2020). Este sería el foco inicial de una pandemia que ha afectado de todo el mundo (WHO, 2020). En Chile, hacia la primera mitad de julio de 2020 el número de casos positivos era de 274.712, de los cuales, 7.262 habían fallecido representando una letalidad de 2,2\% (Ministerio de Salud, 2020).

El COVID-19 infecta las células humanas a través de una de sus glicoproteínas de superficie, la que se adhiere a los dominios de unión del receptor de la enzima convertidora de angiotensina 2, la cual, es abundante en las células alveolares tipo II. Como consecuencia, genera una infección del tracto respiratorio y su gravedad varía desde infección asintomática, leve a severa; de éstos una proporción significativa de pacientes desarrollan una enfermedad crítica como síndrome de dificultad respiratoria aguda (SDRA) y posterior disfunción multiorgánica (Wu \& McGoogan, 2020).

La Sociedad Chilena de Medicina Intensiva, define como sintomatología crítica; el aumento del trabajo respiratorio (uso excesivo musculatura, respiración paradojal,

\footnotetext{
${ }^{1}$ Programa de Doctorado en Ciencias Morfológicas, Universidad de La Frontera, Temuco, Chile.

${ }^{2}$ Centro de Excelencia en Estudios Morfológicos y Quirúrgicos, Universidad de La Frontera, Temuco, Chile.

${ }^{3}$ Unidad de Medicina Física y Rehabilitación. Hospital El Carmen-Dr. Luis Valentín Ferrada, Maipú, Chile.

${ }^{4}$ Departamento de Kinesiología, Universidad Católica del Maule, Talca, Chile.

${ }^{5}$ Laboratorio de Función Ventilatoria Universidad Católica del Maule, Talca, Chile.
} 
alteración de conciencia, hiperadrenergia), taquipnea, hipoxemia refractaria e insuficiencia respiratoria aguda (Sociedad Chilena de Medicina Intensiva, 2020). En este contexto, la ventilación mecánica (VM) aparece como una herramienta fundamental para el manejo de SDRA (Ken et al., 2020). En Chile, el $20 \%$ de los pacientes hospitalizados diagnosticados con infección por COVID-19 han requerido de VM invasiva. Específicamente un 80,6\% de los pacientes en VM permanecen en esta condición por lo menos siete días, y un 66,7 \% más de catorce días (Sociedad Chilena de Medicina Intensiva). Sin embargo, el tratamiento asociado a VM invasiva en pacientes COVID-19, está en estudio y todo aporte contribuiría a comprender de mejor manera esta enfermedad.

En la actualidad la gran mayoría de los ventiladores mecánicos invasivos permiten, a través de su gráfica en tiempo real, conocer la función ventilatoria de pacientes intubados mediante mediciones de mecánica ventilatoria (Araneda et al., 2018). Es así, como a partir de variables de uso habitual en la clínica; presión plateau, PEEP (por sus siglas en inglés positive end expiratory pressure) y volumen tidal $(\mathrm{Vt})$, es posible calcular la presión de distensión (PD) [presión plateau-PEEP] o la distensibilidad [Vt/PD], variables que permitirían predecir, en conjtnto a clínicos y de laboratorio, los tiempos de conexión a VM (Fig. 1B) (Hess, 2014). Por ejemplo, valores de $\mathrm{PD}<15 \mathrm{cmH}_{2} \mathrm{O}$ se asocian con un mejor pronóstico en pacientes con diagnóstico de SDRA en VM (Amato et al., 2015). Situación que se desconoce en el paciente conectado a VM con diagnóstico de COVID-19.

En la conexión a VM, la unidad de cuidado intensivo (UCI) del Hospital El Carmen de Maipú (HEC) en concordancia con las normas internacionales, indica una radiografía de tórax (RT), con el objetivo de controlar la posición del tubo endotraqueal (Protocolo del Paciente Crítico, 2015). Además de esto, la radiografía de tórax permite observar en qué condición se encuentran ambos campos pulmonares. Pese a la utilidad que tiene la RT, su interpretación depende del conocimiento y experiencia de cada profesional (Pragnya et al., 2016). Muchas veces la disponibilidad interpretativa del especialista no es viable, realizándose este ejercicio en condiciones precarizadas y de extrema urgencia. Por tanto, un análisis cuantitativo y estandarizado de la RT, nos permitiría obtener información objetiva y relevante para comprender el compromiso del paciente, independiente de la experiencia de cada profesional (Adejoh et al., 2017).

Así el objetivo de esta investigación fue explorar la relación de las medidas de mecánica ventilatoria y radiográficas con el tiempo de conexión a VM en pacientes COVID-19.

\section{PACIENTES Y MÉTODO}

Realizamos un estudio correlacional, retrospectivo. Desde la ficha clínica electrónica (Florence clínico versión 19.3) y registro kinésico, se obtuvo la siguiente información: i) identificación del paciente, ii) diagnóstico médico, iii) características de la VM y iv) RT. Los meses considerados fueron marzo y abril del año 2020. Los criterios de inclusión fueron; pacientes hospitalizados en el HEC, mayores de edad y con diagnóstico de COVID-19 (confirmado por PCR [+]). Los criterios de exclusión fueron; paciente con enfermedad respiratoria crónica diagnosticada, trastornos músculo esquelético de la columna vertebral y/o tórax y usuarios de VM domiciliaria. Esta investigación se realizó de acuerdo con el Código de Ética de la Asociación Médica Mundial (Declaración de Helsinki) para estudios con seres humanos.

Ventilación Mecánica. Se registró la fecha de inicio y finalización de la VM, donde la diferencia entre ambas fechas fueron los días en VM. Además, se obtuvieron las siguientes variables de mecánica ventilatoria; i) PEEP, ii) Presión plateau, iii) Presión de distensión (Presión Plateau - PEEP) y iv) Compliance estática. Estos datos corresponden a las primeras 12 horas de conexión a VM (Hess)

Radiografía de Tórax. La RT fue tomada por el Tecnólogo Médico de turno con una unidad móvil de radiografía

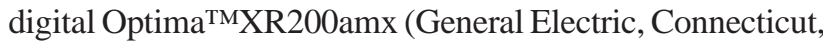
USA) posterior a la intubación orotraqueal, según lo estandarizado por MINSAL. Todas la mediciones se realizaron en el hemitórax derecho , según Bichinho et al., 2010: i) altura retroesternal pulmonar (ARP), desde el punto más alto del ápice pulmonar se proyecta una línea recta hasta la cúpula diafragmática, ii) ancho del pulmón (AP), desde el punto inicial del séptimo espacio intercostal, se proyecta una línea recta hasta el final de la silueta pulmonar contralateral, iii) ángulo costodiafragmático (ACD), primero se localizó el punto del ángulo costodiafragmático, y desde aquí nace una línea que sigue el contorno de la pared torácica lateral y el contorno inicial de la cúpula diafragmática, iv) distancia intercostal (DIC), a partir de línea trazada en la altura retroesternal pulmonar se mide la altura del séptimo espacio intercostal (Fig. 1). El software utilizado para la estimación morfológica de la RT fue PACS, disponible en todas las unidades de evolución del HCM.

Análisis estadístico. Para el análisis estadístico se utilizó el programa estadístico GraphPad Prism (versión 5.0®, San Diego, USA). La distribución de los datos se determinó con la prueba de Shapiro-Wilk. Para establecer la correlación entre las medidas de mecánica ventilatoria-días de VM y 


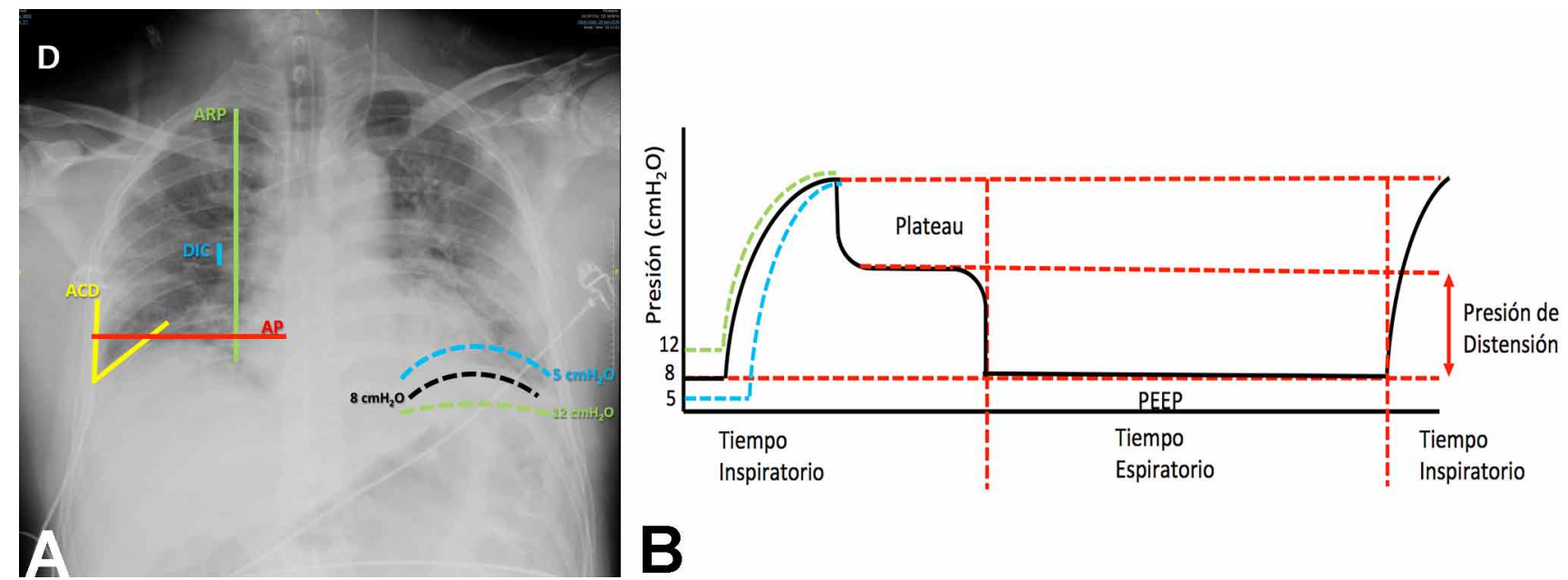

Fig. 1. Mediciones de RT y mecánica ventilatoria en pacientes con COVID-19. A: Medidas obtenidas de la RT: ARP: altura retroesternal pulmonar, AP: ancho del pulmón, ACD: ángulo costodiafragmático, DIC: distancia intercostal. B: Medición de la mecánica ventilatoria: PEEP, plateau, presión de distensión. La figura explica la teorización del mecanismo por el cual el aumento de la presión provoca cambios en la cúpula costodiafragmática. La interacción entre la figura B y A está dada por los distintos valores de $\mathrm{PEEP}\left(5812 \mathrm{cmH}_{2} \mathrm{O}\right.$ ) y como estos repercuten sobre la cúpula costodiafragmática.

radiográficas-días de VM, se utilizó la prueba r de Pearson o Spearman, dependiendo de la distribución de los datos. El nivel de significancia estadística se estableció en un $\mathrm{p}<0,05$.

\section{RESULTADOS}

Del total de pacientes el $61 \%$ era de sexo masculino. El detalle de las medidas radiológicas de tórax y mecánica pulmonar en pacientes con COVID-19 se encuentran en la Tabla I. La PEEP y el plateau se relacionaron significativamente con el tiempo de conexión a VM, r=0,449; $\mathrm{p}=0,035$ y $r=0,472 ; p=0,026$, respectivamente. Del mismo modo, el ACD y la DIC se relacionaron significativamente con el tiempo de conexión a VM, r= 0,462; $\mathrm{p}=0,046$ y r=0,543; $\mathrm{p}=0,009$, respectivamente.

Las relaciones entre días de conexión a ventilación mecánica, medidas de radiografía de tórax y la mecánica ventilatoria en pacientes con COVID-19 se encuentran en la Tabla II.
Los resultados de la relación entre ángulo costodiafragmático, PEEP y plateau en los pacientes se puede observar en la Figura 2.

Tabla I. Detalle de las medidas radiológicas de tórax y mecánica pulmonar en pacientes con COVID-19.

\begin{tabular}{lc}
\hline Variable & Promedio $\pm \mathrm{D}$ \\
\hline Número $(\mathrm{H} / \mathrm{M})$ & $23(14 / 9)$ \\
Edad $(\mathrm{años})$ & $56,82 \pm 12,7:$ \\
Alto $(\mathrm{cm})$ & $16,21 \pm 2,41$ \\
Ancho $(\mathrm{cm})$ & $11,48 \pm 1,34$ \\
Ángulo costofrénico (grados) & $52,09 \pm 6,35$ \\
Distancia intercostal $(\mathrm{cm})$ & $1,19 \pm 0,33$ \\
PEEP $\left(\mathrm{cmH}_{2} \mathrm{O}\right)$ & $10,27 \pm 1,98$ \\
Plateau $\left(\mathrm{cmH}_{2} \mathrm{O}\right)$ & $21,34 \pm 2,73$ \\
Presión de distensión $\left(\mathrm{cmH}_{2} \mathrm{O}\right)$ & $11,27 \pm 1,80$ \\
Compliance $\left(\mathrm{mL} / \mathrm{cm} \mathrm{H}_{2} \mathrm{O}\right)$ & $37,84 \pm 9,90$ \\
Conexión a VM (días) & $11,57 \pm 9,63$ \\
\hline
\end{tabular}

DE: desviación estándar; $\mathrm{H}$ : hombres; M: mujeres; cm: centímetros; PEEP: del inglés, positive end expiratory pressure; $\mathrm{cmH} 2 \mathrm{O}$ : centímetros de agua; $\mathrm{mL} / \mathrm{cmH} 2 \mathrm{O}$ : mililitros partido por centímetros de agua; VM: ventilación mecánica.

Tabla II. Relaciones entre días de conexión a ventilación mecánica, medidas de radiografía de tórax y mecánica ventilatoria en pacientes con COVID-19.

\begin{tabular}{|c|c|c|c|c|c|}
\hline \multicolumn{3}{|c|}{ Radiografía de Tórax } & \multicolumn{3}{|c|}{ Mecánica Ventilatoria } \\
\hline & $\mathbf{r}$ & Valor $p$ & & $\mathbf{r}$ & Valor $p$ \\
\hline Alto $(\mathrm{cm})$ & $-0,187^{p}$ & 0,403 & PEEP $\left(\mathrm{cmH}_{2} \mathrm{O}\right)$ & $0,449 p$ & 0,035 \\
\hline Ancho $(\mathrm{cm})$ & $-0,316^{p}$ & 0,150 & Plateau $\left(\mathrm{cmH}_{2} \mathrm{O}\right)$ & $0,472 \mathrm{~s}$ & 0,026 \\
\hline Ángulo costofrénico (grados) & $0,462^{\mathrm{p}}$ & 0,046 & Presión de distensión & $0,235 p$ & 0,292 \\
\hline Espacio intercostal $(\mathrm{cm})$ & $0,543^{\mathrm{p}}$ & 0,009 & Compliance $\left(\mathrm{mL} / \mathrm{cmH}_{2} \mathrm{O}\right)$ & $0,187 \mathrm{p}$ & 0,403 \\
\hline
\end{tabular}

cm: centímetros; PEEP: del inglés, positive end-expiratory pressure; $\mathrm{cmH} 2 \mathrm{O}$ : centímetros de agua; $\mathrm{mL} / \mathrm{cmH} 2 \mathrm{O}$ : mililitros partido por centímetros de agua; p: Pearson; s: Spearman. 

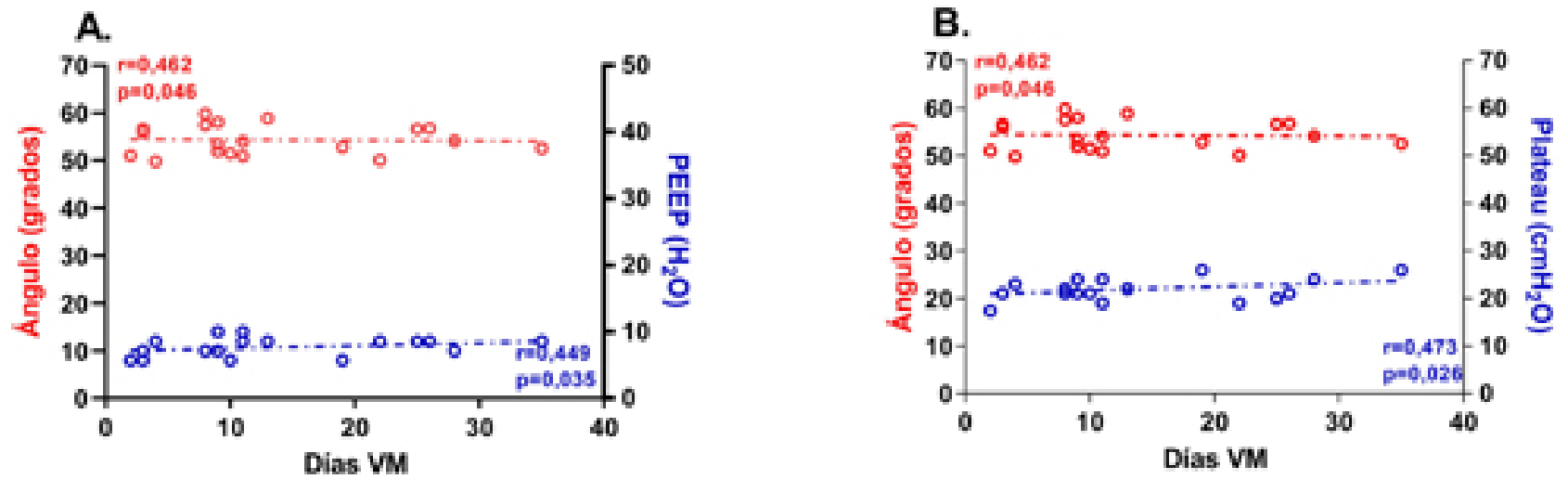

Fig. 2. Relación entre ángulo costodiafragmático, PEEP y plateau en pacientes con COVID-19. PEEP: por sus siglas en inglés positive end expiratory pressure, cmH2O: centímetros de agua. A: Relación Ángulo costodiafragmático-PEEP, con los días de conexión a VM. B: Relación Ángulo costodiafragmático-Plateau, con los días de conexión a VM. Prueba estadística utilizada Pearson.

\section{DISCUSIÓN}

Los principales hallazgos indican que la PEEP y el plateau se relacionaron significativamente con el tiempo de conexión a VM. Por tanto, se puede señalar, que el sistema respiratorio es una estructura interconectada que se mueve coordinadamente (De Troyer \& Wilson, 2016), la cual es sensible a las asistencias externas, en este caso la presión extra entregada por la VM. Con similar comportamiento, el ACD y la DIC también se relacionaron significativamente con el tiempo de conexión a VM.

El estudio refuerza la idea de que el tiempo de conexión a VM invasiva es considerado como un indicador de gravedad de los pacientes en UCI. En relación a esto, los resultados de la presente investigación, concuerdan con los datos reportados en pacientes COVID-19 de Asia, Europa y Estados Unidos. (Ling et al., 2020; Graselli et al., 2020; Graselli et al., 2020; Bhatraju et al., 2020; Cummings et al., 2020). En estos, la media observada fue de 10 (Bhatraju et al., 2020; Graselli et al., 2020) y 11 días (Ling et al., 2020) versus los 11,57 \pm 9,63 días (Tabla I). Este hecho, da cuenta de un comportamiento similar en la evolución del SDRA asociado a la infección por COVID-19 en Chile. De esta manera se concluye que la conexión a VM es proporcional a la evolución de esta patología.

En relación a la mecánica ventilatoria, el uso de un Vt bajo (4-8 $\mathrm{ml} / \mathrm{kg}$ peso ideal), presiones plateau menores a $30 \mathrm{cmH} 2 \mathrm{O}$ y PD por debajo de $15 \mathrm{cmH}_{2} \mathrm{O}$ son, desde antes de esta pandemia, recomendaciones que tienen por objetivo evitar el daño inducido por VM y, por consiguiente, mejorar la sobrevida de pacientes con SDRA (Fan et al., 2017; Amato et al.). Tanto la presión plateau, de 21,34 $\pm 2,73 \mathrm{cmH}_{2} \mathrm{O}$, como la PD, de $11,27 \pm 1,80 \mathrm{cmH}_{2} \mathrm{O}$, observadas en la presente investigación se encuentran dentro de estos límites de seguridad, y son levemente inferiores a las descritas en USA, específicamente Seattle (25 y 13 $\mathrm{cmH}_{2} \mathrm{O}$, respectivamente) (Bhatraju et al.) y Nueva York (27 y $15 \mathrm{cmH}_{2} \mathrm{O}$, respectivamente) (Cummings et al.). Esas investigaciones, también cuantificaron la distensibilidad pulmonar, reportándose valores promedios de $27 \mathrm{~mL} /$ $\mathrm{cmH}_{2} \mathrm{O}$ y $29 \mathrm{~mL} / \mathrm{cmH}_{2} \mathrm{O}$, respectivamente (Cummings et al.; Bhatraju et al.), resultados inferiores a los de esta investigación $\left(37,84 \pm 9,90 \mathrm{~mL} / \mathrm{cmH}_{2} \mathrm{O}\right)$ (Tabla I). Este hecho nos permite comprender la magnitud del compromiso del parénquima pulmonar de la muestra estudiada, la que se manifiesta a través de un aumento de la PD, como traductor de la deformación que experimenta (ingreso de $\mathrm{Vt}$ ) el tejido del sistema respiratorio de estos pacientes (Amato et al.; Cumming et al.). Lo anterior podría vincularse, con el aumento del espacio intercostal, el que es mayor en pacientes con más días de conexión a VM (Tabla II).

La importancia de una adecuada PEEP en pacientes conectados a VM invasiva consiste en; i) lograr reclutar zonas colapsadas de los pulmones, aumentando el espacio disponible para el intercambio gaseoso y ii) controlar el exceso de presión, debido a que valores de PEEP sobre 13 $\mathrm{cmH}_{2} \mathrm{O}$ se asocian a mayor mortalidad en pacientes COVID-19 (Graselli et al.). Así una PEEP alta provoca una sobredistensión de zonas ya aireadas (García-Prieto et $a l ., 2014)$. Lo anterior se traduciría en un ascenso de la presión plateau, y por consiguiente, un aumento de la PD, situación que genera una menor distensibilidad del sistema respiratorio. En relación a los resultados encontrados, 
MUÑOZ-COFRÉ, R.; ARANEDA-MADRID, P.; DEL SOL, M.; ÁLVAREZ-PÉREZ, F.; PÉREZRIQUELME, C.; ESCOBAR-CABELLO, M. \& VALENZUELA-VÁSQUEZ, J. Relación de las medidas de mecánica ventilatoria y radiográficas con el tiempo de conexión a ventilación mecánica en pacientes COVID-19. Un análisis preliminar. Int. J. Morphol., 38(6):1580-1585, 2020.

el valor de la PEEP de los pacientes fue menor $(10,27 \pm$ $1,98 \mathrm{cmH}_{2} \mathrm{O}$ ), en comparación a Cummings et al., $2020 \mathrm{y}$ Graselli et al., 2020 (14 y $\left.15 \mathrm{cmH}_{2} \mathrm{O}\right)$. Pese a esto, la RT indicó un aumento del ACD y de la DIC, paralelo a los valores de la PEEP y plateau (Fig. 2A, B). Esto indica, que la interpretación en conjunto de la RT y la mecánica ventilatoria, permitirían complementar y fortalecer el diagnóstico clínico de la función ventilatoria en pacientes con COVID-19 conectados a VM.

Si bien, la RT es una herramienta de bajo costo y accesible, que permite a través de la observación de zonas de condensación determinar la ocupación alveolar vinculada a la infección por COVID-19 (Wenjing et al., 2020), es poco sensible en estadios tempranos de esta patología (Vernuccio et al., 2020). Sin embargo, en pacientes hospitalizados, la RT puede ser útil para evaluar el progreso de este cuadro. Este principio sería aplicable a la muestra estudiada, la que en su totalidad pertenece a la UCI del HEC. Por otra parte, la evidencia indica que no existen diferencias estadísticamente significativas, con respecto a mortalidad, días de hospitalización y/o en VM, para pacientes en UCI, en la toma de imágenes bajo demanda en comparación con un protocolo de rutina (Rubin et al., 2020; Ken et al., 2020; Wenjing et al.). De este modo, la RT diaria, en pacientes intubados estable COVID-19 no es recomendada (Rubin $e t$ $a l$.$) . En este contexto protocolar, uno de los aportes de la$ presente investigación es, que a partir de la RT de control, además del análisis de rutina (posición del tubo orotraqueal, área de condensación y signos de hiperinsuflación), contribuiría a pronosticar el tiempo de conexión a VM con la medición del ACD y DIC, sumado al análisis de la mecánica ventilatoria (Figura 2A, B). Como proyección, es importante indicar, que la aplicación clínica de estas mediciones es incipiente y debe seguir siendo investigada.

Los resultados obtenidos demuestran que, tanto el ACD como el DIC tuvieron una relación significativa con el tiempo de conexión a VM (Tabla II). La información indica que el aumento de la presión ejercida por una alta PEEP (Tabla I) programada en VM podría disminuir la cúpula diafragmática (Fig. 1) y, por tanto, impactar sobre la estructura de la caja torácica (Hess; Amato et al.). La anatomía y funcionamiento de la caja torácica es reconocida por la interacción coordinada de los músculos encargados de la respiración y cómo esta acción repercute sobre las estructuras óseas que la conforman (De Troyer \& Boriek, 2011). Específicamente, las fibras costales del diafragma van en dirección caudocefálica, desde el reborde inferior de la séptima a duodécima costillas, las cuales al contraerse, elevan y rotan hacia lateral las costillas inferiores. Esta libertad de movimiento se logra, debido a que rotan alrededor del eje de su cuello. Sin embargo, las articulaciones costovertebrales de las costillas 7 a la 10 tienen menos restricción en su movimiento, que las articulaciones costovertebrales de las costillas 1 a la 6 , las que se encuentran unidas al esternón por los cartílagos costales (De Troyer \& Wilson, 2016). Por lo tanto, las costillas superiores tienden a moverse como una unidad con el esternón, situación distinta a lo observado en las costillas inferiores, donde existe una mayor libertad de movimiento. Por tanto, el descenso de la cúpula diafragmática, como resultado de una alta PEEP, aumentaría el ACD y, por contigüidad, también los espacios intercostales (De Troyer \& Boriek; De Troyer \& Wilson). Lo anterior es coincidente con lo reportado en la presente investigación, en la cual, además de encontrar una relación significativa entre ACD y días de VM, también se observó la misma relación entre la DIC y días de VM (Tabla II; Fig. 2A). Ambas consecuencias confirman el paradigma que vincula la unidad operativa de estructura y función.

Por último, esta investigación cuenta con limitaciones que es necesario mencionar; i) por su carácter de investigación retrospectiva, la medición fue obtenida de registros ya estructurados. Además, la ausencia de algunos datos redujo la muestra de estudio, ii) la RT, aunque protocolizada fue tomada por el profesional de turno, existiendo una rotación del equipo de Tecnólogos Médicos, a esto se suma que la imagen fue realizada en pacientes sedados y conectados a $\mathrm{VM}$, por lo que el posicionamiento es una variable que en estudios futuros podría ser mejorada. En conclusión, la presión resultante de la programación del ventilador mecánico se relaciona con el tiempo de conexión a VM. Además, se relaciona a cambios estructurales observados en la RT. En resumen, su interpretación, en conjunto con la RT, deben traducirse en un ajuste personalizado de los parámetros del VM, de acuerdo con la necesidad del paciente y su compromiso ventilatorio.

MUÑOZ-COFRÉ, R.; ARANEDA-MADRID, P.; DEL SOL, M.; ÁLVAREZ-PÉREZ, F.; PÉREZRIQUELME, C.; ESCOBAR-CABELLO, M. \& VALENZUELA-VÁSQUEZ, J. Relations of Ventilatory Mechanical and Radiographic Measures with the ConnectionTime to Mechanical Ventilation in Patients COVID19: A Preliminary Analysis. Int. J. Morphol., 38(6):1580-1585, 2020.

SUMMARY: The lungs are the predominant site of COVID-19 infection. This can lead to severe acute respiratory syndrome (ARDS). In view of its severe symptoms, mechanical ventilation (MV) and its ventilatory mechanics values appear as a fundamental tool. Chest radiography (CR) is a complement to analyze the state of progress of this pathology, although this sometimes depends on the experience of the health team. Thus, the aim of this research was to explore the relationship of ventilatory mechanics and radiographic measures with connection time to MV in COVID-19 patients. Retrospective study, which included 23 patients on MV. Information on ventilatory mechanics variables was collected; PEEP, plateau 
pressure, distension pressure and static compliance. And from CR, lung height and width, costodiaphragmatic angle and intercostal space were measured. The results indicated that ventilatory mechanics variables such as PEEP and plateau were significantly related to connection time to $\mathrm{MV}(\mathrm{r}=0.449 ; \mathrm{p}=0.035$ and $\mathrm{r}=$ $0.472 ; p=0.026$ ), while the radiographic variables Constructed on the basis of the costodiaphragmatic angle and the intercostal space, they showed similar behaviors $(r=0.462 ; p=0.046$ and $r=-0.543$; $\mathrm{p}=0.009)$. In conclusion, the pressure resulting from mechanical ventilator programming, together with the structural changes observed in CR, are related to the connection time to MV.

KEYWORDS; COVID-19; Chest X-ray; Mechanic ventilation.

\section{REFERENCIAS BIBLIOGRÁFICAS}

Adejoh, Th.; Ezeh, H.; Aronu, M.; Nzotta, C. \& Nwefuru, S. A Technique for Appropriate Inferior Collimation in Chest Radiography of Asymptomatic Negroid Adults. West Afr. J. Radiol., 24:52-5, 2017.

Araneda, P.; Améstica, M.; Astorga, E.; Rivero, N.; Robertson, G.; Cruces, P. \& Valenzuela, J. Medición de mecánica ventilatoria y pronóstico de weaning en pacientes pediátricos. XXI Congreso Nacional de Kinesiología 2018, 6-8 de septiembre, La Serena, Chile. doi: 10.13140/RG.2.2.15115.52006.

Amato, M.; Meade, M.; Slutsky, A.; Brochard, L.; Costa, C. \& Schoenfeld, D. Driving Pressure and Survival in the Acute Respiratory Distress Syndrome. N. Engl. J. Med., 372:747-55, 2015.

Bhatraju, P. K.; Ghassemieh, B. J.; Nichols, M.; Kim, R.; Jerome, K. R.; Nalla A. K., et al. Covid-19 in Critically Ill Patients in the Seattle Region - Case Series. N. Engl. J. Med., 382(21):2012-22, 2020.

Bichinho, M. L.; Panizzi, E. A.; Storino G. \& Pinto, D. Analysis of chest radiography of individuals with COPD and its correlation with functional testing. Fisioter. Mov., 25(3):629-37, 2012.

Cummings, M. J.; Baldwin, M. R.; Abrams, D.; Jacobson, S. D.; Meyer, B. J.; Balough, E. M., et al. Epidemiology, clinical course, and outcomes of critically ill adults with COVID-19 in New York City: a prospective cohort study. Lancet, 395:1763-70, 2020.

De Troyer, A. \& Boriek, A. Mechanics of the Respiratory Muscles. Compr Physiol 1:1273-300, 2011.

De Troyer, A. \& Wilson, T. A. Action of the diaphragm on the rib cage. $J$. Appl. Physiol., 121:391-400, 2016.

Fan, E.; Del Sorbo, L.; Goligher, E.C.; Hodgson, C. L.; Munshi, L.; Walkey, A. J., et al. An Official American Thoracic Society/European Society of Intensive Care Medicine/Society of Critical Care Medicine Clinical Practice Guideline: Mechanical Ventilation in Adult Patients with Acute Respiratory Distress Syndrome. Am. J. Respir. Crit. Care Med., 195(9):1253-63, 2017.

García-Prieto, E.; Amado-Rodrígueza, L.; \& Albaiceta G. M. Monitorización de la mecánica respiratoria en el paciente ventilado. Med. Intensiva., 38(1):49-55, 2014.

Grasselli, G.; Zangrillo, A.; Zanella, A.; Antonelli, M.; Cabrini, L.; Castelli, A., et al. Baseline Characteristics and Outcomes of 1591 Patients Infected With SARS-CoV-2 Admitted to ICUs of the Lombardy Region, Italy. JAMA, 323(16):1574-81, 2020.

Grasselli, G.; Greco, M.; Zanella, A.; Albano, G.; Antonelli, M.; Bellani, G., et al. Risk Factors Associated With Mortality Among Patients With COVID-19 in Intensive Care Units in Lombardy, Italy. JAMA Intern. Med., e203539, 2020.

Hess, D. R. Respiratory mechanics in mechanically ventilated patients. Respir. Care, 59(11):1773-94, 2014.
Ken, J. G.; Jolin, W.; Jong-Chie, C. T.; Shin, Y.N.; Sewa, D. W.; Ghee, Ch. P., et al. Preparing your intensive care unit for the COVID-19 pandemic: practical considerations and strategies. Crit. Care, 24:215, 2020.

Ling, L; So, C.; Shum, H. P.; Chan, P. K. S.; Lai, C. K. C.; Kandamby, D. H.; Ho, E., et al. Critically ill patients with COVID-19 in Hong Kong: a multicentre retrospective observational cohort study. Crit. Care Resusc., 22(2):119-25, 2020.

Ministerio de Salud (MINSAL). Informe epidemiológico 31, departamento de epidemiología. Disponible en : https://www.minsal.cl/wp-content/ uploads/2020/07/InformeEPI050720.pdf. [accedido 05-07-2020].

Sociedad Chilena de Medicina Intensiva. Reporte Epidemiológico Clínico mediante Encuesta COVID-19 en Unidades de Pacientes Críticos de Chile. Disponible en: https://www.medicina-intensiva.cl/site/covid/img/ noticias/Reporte_Epidemiologico_Encuesta_COVID_15-05-20.pdf. [accedido 05-07-2020].

Pragnya, M.; Philipsen, R.; Melendez, J.; Scholten, E.; Chanda, D.; Ayles, H., et al. Automatic detection of pleural effusion in chest radiographs. Med. Im. Anal, 28:22-32, 2016.

Protocolo del Paciente Crítico. Intubación y conexión a ventilación mecánica. Unidad de Paciente Crítico. Hospital El Carmen de Maipú.

Rubin, G.; Ryerson, C.; Haramati, L.; Sverzellati, N.; Kanne, J.; Raoof, S., et al. The Role of Chest Imaging in Patient Management During the COVID-19 Pandemic. CHEST, 158(1):106-16, 2020.

Vernuccio, F.; Giambelluca, D.; Cannella, R.; Lombardo, F.; Panzuto, F.; Midiri, M. \& Cabassa, P. Radiographic and chest CT imaging presentation and follow-up of COVID-19 pneumonia: a multicenter experience from an endemic area. Emerg. Radiol., 11:1-10, 2020.

WHO. Brote de enfermedad por COVID-19. Disponible en: https:// www.who.int/es/emergencies/diseases/novel-coronavirus-2019. [accedido 05-07-2020].

Wenjing, Y.; Arlene, S.; Xiaochun, Z.; Guanshu, L.; Zhongzhao, T.; Shihua, Z. \& Minjie, L. The role of imaging in 2019 novel coronavirus pneumonia (COVID-19). Eur. Radiol., 15:1-9, 2020.

Wu, Z.; McGoogan, J. M. Characteristics of and important lessons from the coronavirus disease 2019 (COVID-19) outbreak in China: Summary of a report of 72314 cases from the Chinese Center for Disease Control and Prevention. JAMA, 323(13):1239-42, 2020.

\author{
Dirección para correspondencia \\ Jorge Valenzuela Vásquez \\ Camino a Rinconada \# 1201 \\ Maipú \\ CHILE
}

Email: jorge.valenzuelav@redsalud.gob.cl

Recibido : 16-06-2020

Aceptado: 22-07-2020 